\title{
Diagnostic methods for the etiological assessment of infectious corneal pathology (Review)
}

\author{
MIHAIL ZEMBA $^{1,2^{*}}$, OTILIA-MARIA DUMITRESCU ${ }^{1}$, ANDREEA-ELENA DIMIRACHE $^{1}$, \\ DANIEL CONSTANTIN BRANISTEANU ${ }^{3}$, FLORIAN BALTA ${ }^{2 *}$, MARIAN BURCEA ${ }^{2 *}$, \\ ANDREEA DANA MORARU ${ }^{3}$ and SINZIANA GRADINARU ${ }^{2}$ \\ ${ }^{1}$ Department of Ophthalmology, 'Dr. Carol Davila' Central Military Emergency University Hospital, 010825 Bucharest; \\ ${ }^{2}$ Department of Ophthalmology, 'Carol Davila' University of Medicine and Pharmacy, 050474 Bucharest; \\ ${ }^{3}$ Department of Ophthalmology, 'Grigore T. Popa' University of Medicine and Pharmacy, 700115 Iasi, Romania
}

Received August 6, 2021; Accepted September 7, 2021

DOI: $10.3892 / \mathrm{etm} .2021 .11060$

\begin{abstract}
Infectious keratitis is a leading cause of visual morbidity, including blindness, all across the globe, especially in developing countries. Prompt and adequate treatment is mandatory to maintain corneal integrity and to recover the best possible final visual acuity. Although in most of the cases practitioners chose to employ empirical broad-spectrum antimicrobial medication that is usually effective, in some instances, they face the need to identify the causative agent to establish the appropriate therapy. An extensive search was conducted on published literature before December 2020 concerning the main laboratory investigations used to identify the microbial agents found in infectious keratitis, their indications, advantages, and disadvantages, as well as the results reported by other studies concerning different diagnostic tools. At present, the gold standard for diagnosis is still considered to be the isolation of microorganisms in cultures, along with the examination of smears, but other newer techniques, such as polymerase chain reaction (PCR), next-generation sequencing (NGS), and in vivo confocal microscopy (IVCM) have gained popularity in the last decades. Currently, these newer methods have proved to be valuable adjuvants in making the diagnosis, but technological advances hold promise that, in the future, these methods will have increased performance and
\end{abstract}

Correspondence to: Dr Daniel Constantin Branisteanu or Dr Andreea Dana Moraru, Department of Ophthalmology, 'Grigore T. Popa' University of Medicine and Pharmacy, 16 University Street, 700115 Iasi, Romania

E-mail: daniel.branisteanu@umfiasi.ro

E-mail: andreea-dana.moraru@umfiasi.ro

*Contributed equally

Key words: corneal ulcer, infectious keratitis, bacterial keratitis, fungal keratitis, Acanthamoeba keratitis, polymerase chain reaction, next-generation sequencing, in vivo confocal microscopy availability, and may become the new gold standard, replacing the classic cultures and smears.

\section{Contents}

1. Introduction

2. Corneal scraping, smears, and cultures

3. Corneal biopsy and histopathological examination

4. Molecular assays: Polymerase chain reaction (PCR) and next-generation sequencing (NGS)

5. In vivo confocal microscopy (IVCM)

6. Conclusions

\section{Introduction}

Corneal infections are a major cause of visual morbidity worldwide, resulting in an estimate of up to 2 million unilateral blindness cases per year (1), while also being an important burden on healthcare systems (2). Etiological agents of infectious keratitis can be bacterial, fungal, parasitic, and viral in nature. Important causes of microbial keratitis are trauma, especially that occurring during agricultural work and contact lens wear. While bacterial keratitis is by far the most common form in North America, Europe, Australia, and Oceania, in other parts of the world, especially in tropical climates, fungal causes are equal or greater in frequency compared with bacterial ones (2-4). Microbial keratitis is a medical emergency and timely commencement of appropriate therapy is mandatory to increase the chances of recovery and of preserving a useful visual acuity. Empirical treatment with broad-spectrum antimicrobial medication based on the microbiological profile of each particular region is a frequent practice, supported by the finding that $96 \%$ of isolated microorganisms are sensitive to empirical therapy (5). This practice provides favorable results in the majority of cases, but when therapy fails, occasionally, due to increased resistance of microorganisms to empirical therapy (6), identification becomes mandatory. Although different etiologies differ in their clinical aspect, the identification of the causative agent by clinical examination only, 
even when conducted by experienced cornea specialists, is not correct in a great number of cases (7). Smears and cultures obtained from corneal scrapes remain the gold standard in making the diagnosis. However, even if highly specific, these methods are far from being ideal, as their sensibility is lower than desired for a comprehensive diagnostic tool (2). In recent years, newer techniques have been developed and perfected and come to aid in making the correct diagnosis. Polymerase chain reaction (PCR) is a molecular technique that detects small quantities of microbial deoxyribonucleic acid (DNA) in ocular samples. It is highly sensitive but yields a high rate of false positives (8). However, advances in PCR technology appear promising in their endeavor to increase efficacy and specificity. Next-generation sequencing (NGS) is another molecular method that consists of sequencing the DNA present in a clinical sample and matching the resulting fragments with a DNA database. Although NGS lacks standardization and cannot be used as a single investigation for making the diagnosis, it is of great value as an adjuvant to conventional methods, as it can identify organisms that are new or difficult to isolate in cultures. NGS also assists in the efforts to describe the ocular surface flora, which would help distinguish contaminants from pathogens (9). In vivo confocal microscopy (IVCM) is a non-invasive, rapid assay that allows real-time observation of corneal pathogens at the infection site. It is highly sensitive and specific and has proven particularly useful in diagnosing fungal and Acanthamoeba keratitis (10).

An extensive literature search was conducted in the Medline electronic database, using the PubMed interface. The search process comprised articles written in English, published between May 1992 and December 2020, with the following word combinations 'diagnosis' and, in turn, each of the following: 'corneal ulcer', 'microbial keratitis', 'infectious keratitis', 'corneal infections', 'bacterial keratitis', 'fungal keratitis', 'Acanthamoeba keratitis'. The title and abstract were subsequently evaluated and 51 studies that described and/or compared diagnostic approaches in infectious corneal ulcers, from sampling methods to methods designed for organism identification, were retained. Types of studies considered were cohort, case-control, cross-sectional studies, as well as reviews. Studies of animal models as well as letters to editors, editorials, comments and conference presentations were excluded.

\section{Corneal scraping, smears, and cultures}

When it comes to suspected microbial corneal ulcers, a correct microbiological diagnosis is key to a tailored approach and to increase the chances of healing in cases where therapy proves inefficient. Smears and cultures are the conventional diagnostic methods and are indicated in the following circumstances: i) Corneal infiltrate that is large, central, and/or associated with significant stromal involvement, melting or multiple infiltrates; ii) an infection that is chronic or unresponsive to broad-spectrum antibiotic therapy; iii) clinical features suggestive of non-bacterial keratitis; and iv) history of corneal surgeries (11).

The aim being to discover the actual causative agent(s), every step of the identification process is of great importance, starting with sample collection. The material collected from the infection site must contain enough pathogens to allow identification. Ideally, sample collection should take place before any antimicrobial therapy is commenced, as isolation rates are lower in pretreated cases $(12,13)$. Even though an unsuccessful initial therapy should not impede the identification of the microbial agent (5), usually, antimicrobial therapy is halted $24 \mathrm{~h}$ before sampling, to increase the chance of microorganism recovery (14). Sample collection is usually achieved by performing a 'corneal scrape' from the leading edges and the base of the ulcer (14). Topical anesthesia is used, preferably avoiding tetracaine, as it has an antimicrobial effect. Instruments used for scraping include surgical blades (usually no. 15 blades (15), disposable needles, sterile Kimura platinum spatulas, and calcium alginate swabs moistened in soy trypticase broth $(16,17)$. Sampling is performed using short, firm strokes, applied from the leading margin to the center of the ulceration. If re-scrapes are necessary, the instrument should be either replaced or sterilized again (18). Bensons and Lanier (17) conducted a study that revealed the superiority of calcium alginate swabs moistened in soy trypticase broth over platinum spatulas at yielding positive cultures and recommended using both methods, scraping with the spatula first and then rubbing the ulcer bed with the calcium alginate swab. Other sampling methods that have yielded favorable results are the 'Mini-tip Culturette' (19) and the corneal impression membrane, the latter used in conjunction with transport media and further subculturing in a microbiology laboratory (20).

Smears are obtained by spreading scraped material over clean glass microscope slides, followed by fixation and staining. Standard stains include Gram and Giemsa stains and $10 \%$ potassium hydroxide $(\mathrm{KOH})$ with or without calcofluor white stain $(15,17,18)$. The scraped material should be spread in a thin, even layer, so that elements are not overlapping and can be distinguished from one another, then examined at a microscope at high magnification (usually x400 or more for Giemsa and Gram stains and x200 to x500 for KOH smears) $(11,15,21)$. The Gram stain allows distinction between Gram-positive (which appear purple) and Gram-negative (which appear pink) organisms, while also revealing their shape, grouping, and relation to other components of the smear. $\mathrm{KOH}$ smears appear to be superior to Gram stains in their capability of displaying fungi, Nocardia spp. and Acanthamoeba spp. (18). On the $\mathrm{KOH}$ smears, as cellular debris is cleared, the refractile hyphal fragments of fungi are readily observed. In Gram-stained smears, Nocardia appears as Gram-positive, beaded, thin branching filaments, while in $\mathrm{KOH}$ smears, Nocardia can be recognized as very fine, narrow, intertwined, branching filaments, more easily identifiable. The $\mathrm{KOH}$ wet-mounted stain also displays the characteristic double-walled cysts of Acanthamoeba, which are not always apparent in Gram stains or can be confused with other components of the smear, such as inflammatory cells (18). Specificities of both the Gram and the $\mathrm{KOH}$ smears were over $83 \%$ in most studies $(8,18,21-23)$. In their study, Sharma et al (21) recommended that, if $\mathrm{KOH}+$ calcofluor white smears are positive for fungi, antifungal therapy should be promptly initiated and, if the $\mathrm{KOH}+$ calcofluor white smear is negative for both fungi and Acanthamoeba spp., broad-spectrum antibiotics should be the treatment of choice. The sensitivities and specificities reported by different studies of the Gram and $\mathrm{KOH}$ stains are presented in Table I. The 


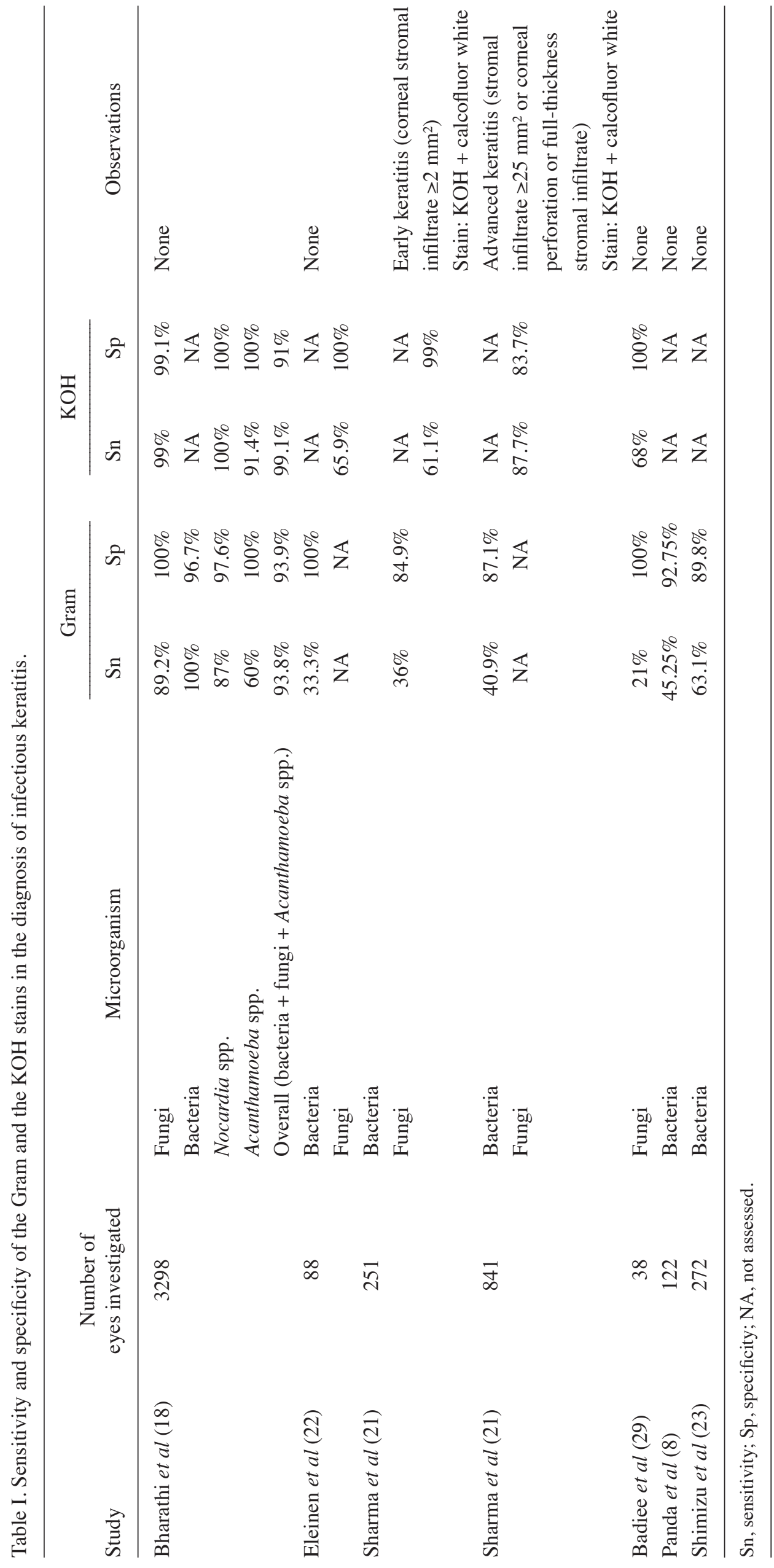


results appear to be influenced by the ulcer dimension, with significantly improved sensitivity if the ulcer size is $>2 \mathrm{~mm}$ in diameter (18). Other factors with potential influence are the amount of scraped material, the accuracy of microscopic examination, and previous antimicrobial therapy $(18,21)$. It was suggested that smear examination is more relevant for therapy than cultures, as the results are more readily available (21). However, the gold standard of diagnosis and the confirmation of smear results reside in cultures (15). Aside from providing certainty regarding the etiology of the keratitis, cultures are the only method that allows testing for antibiotic sensitivity (11).

Cultures are obtained by inoculating the scraped material onto solid or liquid media. Culture material can also consist of contact lenses, their case, and lens solution (11). After sampling, scraped material can be immediately transferred to agar plates (the direct method), or can be sent to a microbiology laboratory with the help of a liquid transport medium, where further subculturing ensues (indirect method) (12). Inoculation is conducted in rows of 'C-streaks' (17) or in a grid pattern (5) at the surface of solid media or by direct swirl into liquid media. Typical solid media include sheep or horse blood agar (BA), chocolate agar (CA) which contains nutrient for the growth of fastidious microorganisms (14), Sabouraud's dextrose agar (SDA) with antibiotics (chloramphenicol and/or gentamycin) for the culture of fungi, non-nutrient agar (NNA) with Escherichia coli (E. coli) overlay for Acanthamoeba spp. $(15,16,18,21)$. In selected cases, special media, such as Löwenstein-Jensen for mycobacteria, and media for anaerobic microorganisms can be used (18). Liquid media are designed for enrichment and growth of organisms, such as, for example thioglycolate medium and Robertson's cooked meat broth, and for transport, the brain heart infusion (BHI) broth and Amies medium are used. Liquid media increase the chance of recovery of the microorganism by diluting the inhibitory factors (24). After inoculation, the plates are incubated (21). Currently, BA, CA, and NNA with $E$. coli overlay are incubated at $36 \pm 1^{\circ} \mathrm{C}$ and examined daily (15). Usually, two BA media are plated, one to be incubated in aerobic, and the other in anaerobic conditions (14). If no growth is observed after 7 days for BA and CA or three weeks for NNA, the plates can be discarded and the culture is deemed negative. Plates with SDA are incubated at $26 \pm 1^{\circ} \mathrm{C}$ and if after 2-3 weeks of daily examination there is no growth, they can be discarded $(15,18)$. Criteria for culture positivity include: i) confluent growth at the inoculation site on solid-phase media; ii) growth of the same microorganism on more than one solid-phase medium; iii) consistency between culture and microscopy findings; and repeated isolation of the same microorganism after different scrapings (18).

Das et al (15) conducted a study that revealed non-inferiority of BA and CA compared with SDA in the detection of fungi. Thus, the authors advocate for the exclusion of SDA when resources are limited. However, further identification of fungi species may require the use of SDA. Bhadange et al (24) investigated the usefulness of liquid media in the diagnosis of infectious ulcers. They found that liquid media are adjuvant to solid media, aiding in the diagnosis of bacterial and, even more so, in that of mixed infections, and suggested that they should be included in standard diagnostic protocols. Kaye et al (12), in an attempt to offer a simplified approach to specimen collection, compared the microbial isolation rates with the direct and indirect method, for the latter using BHI broth as a transport medium. They found no significant difference between the two methods and suggested that the indirect approach could prove useful in cases where the scraped material is in limited quantity and a microbiology laboratory worker would be trained in an improved manner to plate it to all the required media $(12,20)$. The BHI has the additional advantage that it can be maintained at room temperature for $>3$ months. In another study, McLeod et al (25) obtained similar promising results using the Amies medium for transport. However, potential drawbacks of this approach include the delay in isolation, masking of a fastidious microorganism by contaminants or by a more robust pathogen, and inexact colony counting, due to unequal growth of organisms during transport in polymicrobial infections $(24,25)$.

The need to perform smears and cultures in all cases of microbial keratitis was discussed by McLeod et al (5). Their study suggested three possible scenarios. In the first scenario, a safe, but an expensive one, standard smears and cultures are performed in all cases, with the main aim of identifying non-bacterial microorganisms and start appropriate treatment early. The second scenario proposes microbiological assessment only in cases where the history or clinical examination raises the suspicion of a non-bacterial or severe infection (a severe ulcer was defined as one that encompassed the visual axis or involved more than half of the cornea or resulted in corneal thinning to less than half of the normal thickness or was associated with hypopyon). All other cases should receive treatment with broad-spectrum, fortified antibiotics, with close follow-up. According to the third scenario, practitioners should scrape and directly culture all corneal ulcers, then the inoculated plates should be kept in proper conditions in a microbiology laboratory and only be examined if the initial therapy fails. If the initial treatment proves effective, the plates can be discarded without further analysis. However, this requires the maintenance of appropriate supplies by practitioners and collaboration with a microbiology laboratory able to store the cultured plates in the required conditions. In another study, Rodman et al (26) advocated that cultures should be selectively used. On the one hand, cornea specialists, who usually investigate more severe cases, should perform cultures in all patients. On the other hand, general ophthalmologists should use their clinical judgment to decide whether or not cultures are necessary, as small peripheral ulcers generally respond well to empirical therapy, while a tailored therapy guided by cultures is more useful in cases of severe ulcers.

Despite being the gold standard and having good specificity, the sensitivity of smears and cultures is far from ideal. Culture sensitivities range between 32.7-79.4\% (2). Moreover, cultures may take several days until the results are ready. This delays appropriate therapy in a condition where time is vision. Smears sensitivities lie in the interval 27.3-61.6\% (2). A previous study reported higher sensitivity of smears compared with cultures probably because, as cultures require living microorganisms, the examination of smears can identify both viable and non-viable ones (22). Possible explanations for the low sensitivity of smears and cultures may reside in pretreatment with antibiotics, the small size of the samples, and the difficulty in optimally incubating the plates $(2,12)$. This explains the ongoing search for an optimized method with higher sensitivity along with a lower rate of false-positive results. 


\section{Corneal biopsy and histopathological examination}

The corneal biopsy is another valuable tool in cases where there is a failure of the initial therapy. A corneal biopsy is not without risks as there is a possibility of corneal perforation and of scarring or irregular astigmatism with a significant effect on visual outcome (16). As a result, a correct indication is mandatory.

The main indications for performing a corneal biopsy are: i) the clinical progression of the infection or lack of response to treatment despite the topical antimicrobial therapy, both in the setting of persistently negative microbiological results and in cases with positive corneal scrapings, but when the additional role of another unidentified microorganism is suspected; and ii) a progressive infiltration in the corneal stroma with an intact epithelium, where corneal scrapes are ineffective $(16,27,28)$.

The corneal biopsy provides larger and more profound tissue than corneal scrapes, improved to perform both microbiological (smears and cultures) and histopathological examinations. A corneal biopsy is unique compared with other sampling methods allowing histopathological examination. The histopathological examination has the advantage of a quicker response (often within $72 \mathrm{~h}$ ), being of great value in identifying organisms that are either difficult or slow to culture. Moreover, a secondary aim of the procedure resides in excising infected and necrotic tissue, which assists in the healing process (16). The corneal button resulted after therapeutic penetrating keratoplasty, can also undergo histopathological examination (29). Before the biopsy, antimicrobial therapy should be withheld for at least $24 \mathrm{~h}(16,28)$. The procedure may be performed at the slit lamp, in a minor procedure room, or in the operating room under topical anesthesia $(16,27,28)$, although some patients may require a retrobulbar block (27). The technique usually employed is a lamellar corneal biopsy, which is performed using either a sterile disposable $2-\mathrm{mm}$ or 3 -mm dermal trephine and/or a surgical blade. The sample should contain both clinically affected cornea and a portion of the adjacent unaffected cornea at the leading edge of the ulcer $(16,27,28)$. If possible, the visual axis should be avoided (16). New techniques have also emerged. Kim et al (30) described the possibility of performing the biopsy with the use of a femtosecond laser. Diamond et al (31) devised a new blade with the ability to accumulate a large volume of tissue which, combined with subsequent micro-homogenization, yielded high culture positivity $(71 \%)$, which, however, did not prove statistically significant. The biopsy specimen is then placed in a balanced salt solution and sent to the microbiology laboratory, where it undergoes homogenization with sterile trypticase soy broth with the use of a mortar and pestle. The mixture is then inoculated into the appropriate media $(27,28)$.

If a histopathological examination is desired, the specimen can be bisected, with one half being placed in $10 \%$ buffered formalin and sent to a pathology laboratory (28). For the histopathological examination, the material is processed for paraffin embedment (28), cut into 3-mm (16) or 5-mm (28) slices with the microtome, and then stained. Routine stains include hematoxylin-eosin (H\&E), Gram stain for bacteria, Gomori methenamine silver (MethAg), and periodic acid Schiff (PAS) for fungi, Ziehl Neelsen, Fite special stain, and the auramine fluorescent stain for mycobacteria (16). The positivity of the herpes simplex virus is demonstrated by its cytopathic effects observed in the epithelial cells at the margin of the ulcer, such as multinucleation, molding, and margination (16). Alexandrakis et al (27) conducted a study on 33 patients aimed at establishing the utility and indications of corneal biopsy. A total of $82 \%$ of the corneal biopsies were positive by either microbiological or histopathological examination of the biopsy material. Other studies, such as that of Robaei et al (16) and that of Younger et al (28) reported lower rates of biopsy positivity of 39 and $42 \%$, respectively. However, studies had similar findings concerning the discovery of a new microorganism [81\% (27) and 73\% (16)] and the need for changing the initial therapy after obtaining the results [89\% (27) and $65 \%$ (28)], which occurred in most of the biopsy-positive patients. When used correctly, the corneal biopsy is a valuable investigation that can identify a new pathogen, not detected by previously used diagnostic methods, and lead to a case-saving change in therapy. It should, however, be used cautiously, due to its potential risks. Corneal biopsy and histopathological examination also allow, in cases with less typical clinical aspect, to exclude ocular involvement from other systemic diseases such as Lyell syndrome, Stevens-Johnson syndrome, lichen planus $(32,33)$, or Rowell syndrome (34).

\section{Molecular assays: Polymerase chain reaction and next-generation sequencing}

PCR is a sensitive and rapid molecular method used to amplify and render detectable minute quantities of microbial DNA in pathological specimens. It is of particular use in cases with little available specimen quantity and when there is a scarcity of pathogens in the specimens. This renders it an attractive tool in the diagnosis of ocular infections and PCR has been successfully used in the diagnosis of uveitis, endophthalmitis, as well as of viral ocular infections $(8,23,30)$. Corneal material for the PCR assay is obtained by corneal scraping with the same method as aforementioned. Following sampling, the specimen is placed in a lysis PCR buffer and kept at a low temperature until further processing (8). DNA extraction is performed to separate DNA from proteins, cell membranes, and components. This can be performed manually or using commercially available kits (35). DNA extraction can be omitted, as revealed by Zhao et al (36). After DNA extraction, a PCR mix is prepared by adding the reagents separately or by using a master mix, which contains the necessary ingredients. The reaction mixture contains the template DNA, the four nucleotides in equal proportions, two primers, one forward and one reverse, a buffer, and the Taq-DNA polymerase (37). Primers are 18-25 base pair (bp) long single-stranded DNA pieces that must match the sample DNA and that represent the starting points for strand synthesis. Primers may be specific for a certain microorganism or can target sequences that are common for more microorganisms such as the 16S ribosomal ribonucleic acid (rRNA) gene for bacteria and the 18S rRNA gene for fungi $(8,23)$. Subsequently, the mixture is placed in a thermocycler, where repeated cycles consisting of three basic steps, template denaturation, primer annealing, and new strand extension, take place. Before the first cycle, an initial denaturation can be performed. During each cycle, the temperature is rapidly raised to $92-96^{\circ} \mathrm{C}$ for $15-60 \mathrm{sec}$ and the two DNA 
strands dissociate (denaturation). The mixture is then cooled to reach a temperature of $42-75^{\circ} \mathrm{C}$ for primer annealing. The third step, extension, requires a temperature of $72-74^{\circ} \mathrm{C}$, at which the Taq polymerase adds 35-100 nucleotides per second to complete the complementary strand. Each cycle is stopped by raising the temperature to $92^{\circ} \mathrm{C}$. Usually, between 25 and 40 cycles are necessary (35). In the end, a final 5-7 min of extension at $72^{\circ} \mathrm{C}$ can be employed. The result of the reaction, the amplified material, is then electrophoresed in agarose gel, then examined using ultraviolet light or colored with ethidium bromide (38) and compared with a marker of known bp length $(8,22)$. Most investigations begin with a broad-range PCR, with primers that are either bacterium- or fungus-specific $(22,23,29,38,39)$. This allows differentiation between bacterial and fungal keratitis, which is critical to therapy, as numerous clinicians are hesitant, especially when the history and the clinical aspect are not very suggestive, to initiate antifungal therapy, fearing its potential toxicity. Further identification can be performed either by using more specific primers $(29,40)$ or by performing DNA sequencing $(22,23,38,39)$, which also helps in determining real pathogens from contaminants. (22) However, DNA sequencing methods are not routinely used, because they are time-consuming, usually requiring 5 to 7 days (3), not cost-effective $(8,40)$, and also yield numerous false-positive results (8).

PCR has some advantages that render it superior to smears and cultures in the detection of pathogens. Firstly, it provides quick results, usually within $2-8 \mathrm{~h}$ (compared with a minimum of $24-48 \mathrm{~h}$ in the case of cultures) $(8,40)$, therefore permitting the prompt choice of appropriate therapy based on the result. Secondly, the method requires a small amount of tissue, which is helpful considering the small volume of samples usually available from corneal scrapes. Most importantly, it requires very little pathological DNA, one copy of DNA being enough to detect the pathogen (8). The result of these qualities is a high detection rate, PCR being a method with high sensitivity. PCR proves useful in culture-negative cases and in cases with fastidious or hard-to-culture microorganisms (23). PCR also displayed positivity for organisms that were not previously regarded as responsible for infectious keratitis $(3,39)$. This raises the hypothesis that some culture-negative cases are caused by novel or atypical microorganisms that are difficult to grow in cultures. Further investigation, including sequencing, is required in such cases to better characterize these organisms and to understand if they are indeed the causative agents (3). However, PCR lacks the high specificity of smears and cultures and has an important rate of false-positive results, as microorganisms belonging to ocular flora, tears, or laboratory contaminants can be amplified in the reaction and considered as causative agents $(3,8,40)$. PCR can detect both viable and non-viable microorganisms $(3,8)$. This can be both an advantage and a drawback, as, on the one hand, PCR can demonstrate infection in culture-negative cases, but, on the other hand, it cannot differentiate between active and inactive infections. As such, all PCR results should be judged in a clinical context. An important limitation of PCR consists in the fact that the primer selection process involves a pretest suspicion regarding the etiologic agent and, thus, PCR can miss the diagnosis if this selection is incorrect. Primer selection also prevents direct PCR from specifically detecting the exact pathogen in every case, as only the preselected organisms can be detected (9). Other disadvantages comprise its high cost, low availability, and the impossibility to test antibiotic susceptibility (8).

There are numerous variants to the classic, direct PCR. Multiplex PCR permits amplification of more than one target at a time, thus allowing analysis and identification of multiple microorganisms in the same PCR cycle. Nested PCR comprises two consecutive PCR reactions, in which the result of the first reaction is used as a target for the second reaction (35). This technique has increased sensitivity, but should not be routinely used, as it has a high rate of false positives (8). Real-time PCR uses special dyes to estimate the quantity of the product in the sample as the amplification progresses (35). Real-time PCR deals with some of the drawbacks of classic PCR. It assesses how the number of DNA copies in the sample varies over time and helps in monitoring the course of the disease and, being a quantitative method, it allows discrimination between contaminants and actual pathogens $(8,40)$. Most studies report high PCR sensitivities, of over $85 \%$, in bacterial and fungal keratitis $(8,22,36,38,39)$, except for Nocardia spp. (3). PCR appears to be much more sensitive than cultures and smears, as observed in Table II. Itahashi et al (40), who used real-time PCR with a cycling probe, which appears to yield improved results compared with the linear and structural probes, reported $100 \%$ sensitivity in 5 out of the 6 pathogens simultaneously tested for. This, however, was not the case in other studies. Shimizu et al (23) conducted a study aimed at evaluating the usefulness of the quantitative results provided by real-time PCR for the $16 \mathrm{~S}$ rDNA gene in the diagnosis of bacterial keratitis. Results revealed that a number of copies greater than $10^{3}$ favored the diagnosis, while $100 \%$ specificity was reached at a copy number of $6.9 \times 10^{4}$. PCR as a sole investigation did not demonstrate significant superiority to cultures and smears, with a reported sensitivity of $63.6 \%$. However, when used alongside cultures and smears, PCR significantly improved diagnostic efficacy. PCR and culture results were concordant in most cases. Furthermore, in culture-negative cases, PCR also yielded a smaller number of copies, while pretreatment with antibiotics resulted in both a lower yield at the 16S rDNA real-time PCR and more culture-negative results. Concordance rates of PCR and culture results vary widely, ranging from 43 to $93 \%(3,22,36,38-40)$, with higher agreement rates in cases of fungal keratitis compared with bacterial keratitis $(3,22)$, probably because bacteria are more common than fungi in the ocular flora and, therefore, may more commonly become contaminants (3). PCR helps identify pathogens in culture-negative cases, being positive in $53.7-88 \%$ of such cases $(3,22,23)$. However, the specificity and positive predictive value of PCR are imperfect (Table III), usually lower than those of conventional methods $(8,23,29,36)$. The high rate of false positives is perhaps the most important reason why most authors, while acknowledging the usefulness of PCR as an adjuvant in the diagnosis, still consider cultures to be the gold standard in providing the diagnosis of infectious corneal ulcers. New advances in PCR assessment aim to increase sensitivity, specificity and help differentiate between different microorganisms. Goldschmidt et al (41) used High Resolution Melting real-time PCR (PCR-HRM) to devise a test that could differentiate between filamentous fungi and yeasts, 
Table II. Comparative sensitivities of smears, cultures and PCR in different studies.

\begin{tabular}{|c|c|c|c|c|c|c|c|c|}
\hline \multirow[b]{2}{*}{ Study } & \multirow{2}{*}{$\begin{array}{l}\text { Number of } \\
\text { eyes } \\
\text { investigated }\end{array}$} & \multirow[b]{2}{*}{ Microorganism } & \multirow[b]{2}{*}{ PCR type } & \multirow[b]{2}{*}{ Target } & \multirow{2}{*}{$\begin{array}{l}\text { Smear } \\
\text { type }\end{array}$} & \multicolumn{3}{|c|}{ Sensitivity } \\
\hline & & & & & & Smear & Culture & PCR $(\%)$ \\
\hline Eleinen et al (22) & 88 & Bacteria & Direct PCR & 16S rRNA gene & Gram & $33.33 \%$ & $57.33 \%$ & 87.88 \\
\hline Eleinen et al (22) & 88 & Fungi & Direct PCR & 18S rRNA gene & $\mathrm{KOH}$ & $65.91 \%$ & $59.09 \%$ & 90.91 \\
\hline Badiee et al (29) & 38 & Fungi & Nested PCR & 18S rRNA gene & $\begin{array}{l}\mathrm{KOH} \\
\text { Gram }\end{array}$ & $\begin{array}{l}68 \% \\
21 \%\end{array}$ & $57 \%$ & 75 \\
\hline Panda et al (8) & 122 & Bacteria & Direct PCR & 16S rRNA gene & Gram & $45.25 \%$ & NA & 86.96 \\
\hline Shimizu et al (23) & 272 & Bacteria & Real-time PCR & 16S rRNA gene & Gram & $63.1 \%$ & $51.8 \%$ & 63.6 \\
\hline Zhao et al (36) & 67 & $\begin{array}{l}\text { Bacteria } \\
\text { Fungi } \\
\text { Acanthamoeba } \\
\text { spp. }\end{array}$ & $\begin{array}{l}\text { Direct PCR } \\
\text { without } \\
\text { template } \\
\text { DNA } \\
\text { extraction }\end{array}$ & $\begin{array}{l}\text { 5.8S rRNA gene } \\
16 \mathrm{~S} \text { rRNA gene } \\
\text { The conservede } \\
29 \text { region of } \\
\text { 18S rRNA gen } \\
\text { The US4 region } \\
\text { of the envelope } \\
\text { glycoprotein } \mathrm{G}\end{array}$ & $\begin{array}{l}\text { Gram + } \\
\mathrm{KOH}\end{array}$ & NA & $47.1 \%$ & 81.8 \\
\hline
\end{tabular}

NA, not assessed.

Table III. Specificity and positive predictive values of PCR reported by different studies.

\begin{tabular}{|c|c|c|c|c|c|c|}
\hline Study & $\begin{array}{c}\text { Number of } \\
\text { eyes investigated }\end{array}$ & Microorganism & PCR type & Primer target & Specificity (\%) & $\begin{array}{c}\text { Positive } \\
\text { predictive } \\
\text { value (\%) }\end{array}$ \\
\hline Badiee et al (29) & 38 & Fungi & Nested PCR & 18S rRNA gene & 70 & 50 \\
\hline Panda et al (8) & 122 & Bacteria & Direct PCR & 16S rRNA gene & 86.96 & 83.93 \\
\hline Shimizu et al (23) & 272 & Bacteria & Real-time PCR & 16S rRNA gene & 67.5 & 44.9 \\
\hline \multirow[t]{6}{*}{ Zhao et al (36) } & 67 & Bacteria & Direct PCR & 5.8S rRNA gene & & \\
\hline & & Fungi & without & 16S r RNA gene & & \\
\hline & & Acanthamoeba spp. & template & The conserved 29 & & \\
\hline & & & DNA & region of $18 \mathrm{~S}$ & & \\
\hline & & & extraction & rRNA gene & & \\
\hline & & & & $\begin{array}{l}\text { The US4 region of } \\
\text { the envelope } \\
\text { glycoprotein G }\end{array}$ & 81.8 & 96.2 \\
\hline
\end{tabular}

as well as among the most important pathogenic species, to aid in treatment choice. For cases of Acanthamoeba spp., special assays have been developed, such as the 'Qvarnstrom' and 'Riviere' assays. Karsenti et al (42) designed a novel TaqMan primer-probe set for Acanthamoeba detection, that showed promising results, with a sensitivity of $100 \%$, a specificity of $94 \%$, a positive predictive value of $82.8 \%$, and a negative predictive value of $100 \%$. Overall, PCR is a valuable addition to smears and cultures in the diagnosis of infectious keratitis. However, to become a comprehensive diagnostic method that could replace the conventional approaches, it still requires improvements to increase specificity, reduce the rate of false positives, establish protocols and diagnostic thresholds, increase availability and reduce the cost.
NGS is a metagenomic assay, in which millions of small (150-500 bp in length) DNA fragments are simultaneously sequenced in a matter of hours (9). In cases of infectious keratitis, sample tissue is often represented by a corneal biopsy or penetrating keratoplasty buttons, which are then formalin-fixed paraffin-embedded (43). Most of the sequenced DNA is of human origin, while pathogenic DNA is found, on average, in $1.7 \%$ of the readings (9). NGS is divided into two strategies: Targeted amplicon sequencing (TAS), which uses PCR as a starting point, and microbial whole-genome sequencing (MWGS), which sequences fragments found throughout the microbial genome. While TAS is more cost-effective and useful at identifying certain pathogens of interest, MWGS has the advantage of unbiased sequencing and is, thus, valuable in 
detecting new or atypical pathogens. Resultant sequences are then compared with databases of microbial DNA with the help of metagenomic classification engines, such as Centrifuge and Kraken, to identify if they match any known pathogen $(9,43)$. The assay usually requires at least 3.5-4 days, which makes it comparable to cultures (9). In a study by Li et al (43), NGS correctly identified bacteria and Acanthamoeba spp. in $100 \%$ of cases, fungi in $66 \%$ of cases, and herpetic keratitis in $33 \%$ of cases. NGS could, therefore, become very valuable in culture-negative cases with atypical or new pathogens and could also help in describing the normal ocular surface flora. Another study by Holmgaard et al (44) compared an NGS-based 16S-18S rRNA gene assay with real-time PCR in the detection of non-viral causes of infectious keratitis. Having real-time PCR as the standard for comparison, the NGS-based assay provided favorable results, with a sensitivity of $88 \%$ and a specificity of $100 \%$. Discrepancies between NGS and culture results may indicate a possible role for NGS as a complementary method, especially in cases unresponsive to treatment $(45,46)$. Nevertheless, for now, several improvements are necessary before making NGS a routine diagnostic method. Firstly, DNA databases must be enriched and the process of searching improved. Secondly, thresholds for the number of sequences necessary to establish the diagnosis of infection must be determined for each pathogen, to exclude contaminants. For this purpose, characterization of the corneal microbiota is required. Furthermore, easier to obtain corneal samples, such as corneal scrapes, may be needed (43). Even if, for the time being, cultures are essential for diagnosis, NGS appears promising, a complementary method to conventional assays and future advances may lead to the development of a more accessible and reliable test, that could replace classic diagnostic tools.

\section{In vivo confocal microscopy (IVCM)}

IVCM is a rapid, repeatable, non-invasive examination that allows real-time visualization of corneal layers and structures, as well as of potential pathological agents (10). Possible situations when one should consider referral for IVCM are: i) current treatment with antifungal or anti-Acanthamoeba spp. therapy, which results in a sterile anterior corneal stroma, while actively proliferating microorganisms are found in the profound, inaccessible stroma; ii) deeply situated infiltrates, where corneal scrapes do not have access and which would otherwise require corneal biopsy; and iii) microbial keratitis occurring after corneal surgery, such as intracorneal implants and refractive surgery (47).

The particularity of IVCM lies in the fact that it is designed to eliminate light scatter from elements that lay outside the focal plane. It does so by using a system of pinhole apertures and objective lenses, which ensure that the point light source is matched by or is confocal with the detector (48). The result is, in newer microscopes, an up to x500 magnification, and a high contrast image, consisting of thin coronal sections of the cornea $(10,47)$. Corneal layers of interest comprise the epithelial and subepithelial layers, the subbasal nerve plexus, and different levels of depth of the stroma (10). Image acquisition is usually performed in an anterior to posterior fashion, beginning with the central part of the ulcer or infiltrate and then moving towards the periphery of the lesion, in such a way that all areas are scanned (49). There are multiple acquisition modes, such as single sections at a certain depth, volumes (serial images spanning over an interval of depth), and sequence scans (video composed of scans at a defined depth) (49). Microscopes currently in use for performing IVCM are the tandem scanning confocal microscope, which is no longer produced, the slit-scanning confocal microscope (e.g., the Confoscan 4 produced by NIDEK Technologies SRL), and the laser scanning confocal microscope, the newest type, with high magnification and axial resolution, represented by Heidelberg Retinal Tomograph 3 (HRT3), used alongside the Rostock Cornea Module (RCM), which together make the HRT3/RCM produced by Heidelberg Engineering, Germany (4). IVCM is a contact assay using an immersion lens and, therefore, requires topical anesthesia, a coupling agent, and a disposable sterile cap that is applied to the ocular surface (50). Examination of the captured images can demonstrate, in cases of infectious keratitis, inflammatory cells and characteristic aspects of different microorganisms, especially fungi and Acanthamoeba spp. Bacteria are too small to be visualized, thus in bacterial keratitis, the images show an abundance of neutrophils, dendritic cells, and lymphocytes, but no atypical microorganisms (51). Filamentous fungi appear as hyperreflective, linear opacities with a diameter of 3-8 $\mu \mathrm{m}, 200-400 \mu \mathrm{m}$ long, and irregular branching. Differential diagnosis with corneal nerves is warranted. Yeasts appear as round or oval, hyperreflective, with a length of 10-40 $\mu \mathrm{m}$ and may develop pseudohyphae (52). IVCM can identify both forms of Acanthamoeba spp. Cysts are double-walled, highly reflective, with a diameter of $15-28 \mu \mathrm{m}$, while trophozoites, the active form, are hyperreflective, oval, or irregular in shape, with a diameter of $25-40 \mu \mathrm{m}$ and surrounded by edema, which is hyporeflective $(50,52)$. IVCM is of great use in cases of fungal or Acanthamoeba keratitis, being highly sensitive and specific, as observed in Table IV. In cases of Acanthamoeba keratitis, reported sensitivity is usually $>80 \%$, while specificity exceeds $84 \%$. In cases of fungal keratitis, the majority of studies reported rates of sensitivities of $>85 \%$ and specificities of $>90 \%(10,47,49,50)$. Positive predictive values were higher than $95 \%(47,49)$. However, Wang et al $(10)$ reported an IVCM sensitivity at detecting fungal keratitis of only $66.7 \%$ but attributed this to the small number of patients with fungal keratitis in their study. Goh et al (49) compared IVCM with cultures and real-time PCR (the Riviere assay) in cases of suspected Acanthamoeba keratitis and found that IVCM had the highest sensitivity of the three. Moreover, IVCM was positive not only in all the positive culture and PCR cases, but also showed positivity in cases where the other two assays were negative. The high sensitivity and specificity render IVCM a valuable adjuvant to the other diagnostic assays and some authors advocate for its use early in the course of the disease (49). As it yields rapid results, it allows the prompt beginning of appropriate treatment. This is crucial for a favorable prognosis, especially because most ophthalmologists are hesitant to start antifungal and anti-Acanthamoeba medications without a positive diagnosis as the therapy is usually toxic and requires long periods. IVCM also helps appreciate the depth of the infection in the corneal stroma, which is an important prognostic factor $(4,47)$. Moreover, being non-invasive, IVCM can be repeated during the treatment, thus also being useful 
Table IV. Sensitivity and specificity of IVCM in different studies.

\begin{tabular}{|c|c|c|c|c|}
\hline Study & Number of eyes investigated & Microorganism & Sensitivity (\%) & Specificity (\%) \\
\hline Goh et al (49) & 25 & Acanthamoeba spp. & $88.9-100$ & 100 \\
\hline Kanavi et al (50) & 133 & & 100 & 84 \\
\hline Vaddavalli et al (47) & 148 & & 80 & 100 \\
\hline Wang et al (10) & 49 & & 91.7 & 100 \\
\hline Kanavi et al (50) & 133 & Fungi & 94 & 78 \\
\hline Vaddavalli et al (47) & 148 & & 89.2 & 92.7 \\
\hline Wang et al (10) & 49 & & 66.7 & 100 \\
\hline Vaddavalli et al (47) & 148 & $\begin{array}{l}\text { Fungi }+ \\
\text { Acanthamoeba spp. }\end{array}$ & 88.3 & 91.1 \\
\hline Wang et al (10) & 49 & & 85.3 & 100 \\
\hline Wang et al (10) & 49 & Bacteria & 66.7 & 100 \\
\hline Wang et al (10) & 49 & Viruses & 100 & 100 \\
\hline
\end{tabular}

in monitoring the therapy (47). IVCM has its disadvantages. Patient collaboration and patience are required during testing as different areas of the corneal ulcer and surrounding cornea are scanned at different depths and using different scanning modes (50). In cases of uncooperative patients, there is a higher rate of false-negative results. Higher false-negative results are also yielded by excessively hazy corneas or by an abundance of inflammatory cells that mask the characteristic elements of pathogens (10). Moreover, IVCM is user-dependent and requires experience for both acquiring and interpreting images (4). Using experienced users, Vaddavalli et al (47) and Wang et al (10) reported substantial intra-observer agreement rates, with Cohen's kappa values of 0.6 and 0.68 , respectively, while the intra-observer agreement was very favorable, with kappa values of 0.795 and 0.94 , respectively. However, the need for experienced users in conjunction with its high cost results in a low availability (47). As such, for the moment, IVCM cannot be used alone, but alongside cultures and smears.

\section{Conclusions}

While recent years have seen the development of numerous new diagnostic tools that have been successfully used in cases of infectious corneal ulcers, at present, the gold standard for making the diagnosis still resides in cultures. Cultures are highly specific and allow testing for antibiotic susceptibility. Even though cultures require a longer time than other methods until results are ready and their sensitivity is lower than desired, they still provide the diagnosis confirmation and the reference standard for the evaluation of all the other methods. If positive, smears can facilitate diagnosis and allow prompt initiation of appropriate therapy. IVCM, when accessible, is a non-invasive, rapid tool, with high specificity and sensitivity, of great value especially in cases of fungal and Acanthamoeba keratitis. Molecular methods, consisting of PCR and NGS, are rapidly evolving and improving and are of particular use in culture-negative cases, as well as in infections with atypical or novel organisms. They are highly sensitive but yield a high rate of false positives. Advances in technology are promising, as an improved understanding of both the commensal and the pathological organisms found on the ocular surface, as well as their interactions with each other and with the human host, is observed. In time, as molecular methods gain promptness, specificity, and sensitivity, the replacement of conventional methods by these new, improved assays may be observed.

\section{Acknowledgements}

Not applicable.

\section{Funding}

No funding was received.

\section{Availability of data and materials}

Not applicable.

\section{Authors' contributions}

MZ, DCB, MB, and FB contributed to the design of the study, participated in the entire review process, and prepared the manuscript. MZ and OMD confirm the authenticity of all the raw data. OMD, ADM, SG, and AED contributed to the literature research, and the analysis and critical interpretation of the data. MZ and DCB conceived the study and revised the manuscript. All authors read and approved the final version of the manuscript.

\section{Ethics approval and consent to participate}

Not applicable.

\section{Patient consent for publication}

Not applicable.

\section{Competing interests}

The authors declare that they have no competing interests. 


\section{References}

1. Whitcher JP, Srinivasan M and Upadhyay MP: Corneal blindness: A global perspective. Bull World Health Organ 79: 214-221, 2001.

2. Ung L, Bispo PJM, Shanbhag SS, Gilmore MS and Chodosh J: The persistent dilemma of microbial keratitis: Global burden, diagnosis, and antimicrobial resistance. Surv Ophthalmol 64: 255-271, 2019.

3. Kim E, Chidambaram JD, Srinivasan M, Lalitha P, Wee D, Lietman TM, Whitcher JP and Van Gelder RN: Prospective comparison of microbial culture and polymerase chain reaction in the diagnosis of corneal ulcer. Am J Ophthalmol 146: 714-723, 723.e1, 2008

4. Kumar RL, Cruzat A and Hamrah P: Current state of in vivo confocal microscopy in management of microbial keratitis. Semin Ophthalmol 25: 166-170, 2010

5. McLeod SD, Kolahdouz-Isfahani A, Rostamian K, Flowers CW, Lee PP and McDonnell PJ: The role of smears, cultures, and antibiotic sensitivity testing in the management of suspected infectious keratitis. Ophthalmology 103: 23-28, 1996.

6. Grecu C, Grecu A, Şerban IL,Hurjui I,Delianu C, Mărănducă MA Popovici D, Grădinaru I, Mitrea M and Hurjui LL: Prevalence of nasal carriage of Staphylococcus aureus with special reference to number of methicillin resistance and antimicrobial evaluation among apparently people with good health status. Rev Med Chir 122: 819-825, 2018.

7. Dalmon C, Porco TC, Lietman TM, Prajna NV, Prajna L, Das MR, Kumar JA, Mascarenhas J, Margolis TP, Whitcher JP, et al: The clinical differentiation of bacterial and fungal keratitis: A photographic survey. Invest Ophthalmol Vis Sci 53: 1787-1791, 2012

8. Panda A, Pal Singh T, Satpathy G, Wadhwani M and Matwani M Comparison of polymerase chain reaction and standard microbiological techniques in presumed bacterial corneal ulcers. Int Ophthalmol 35: 159-165, 2015.

9. Ma L, Jakobiec FA and Dryja TP: A review of next-generation sequencing (NGS): Applications to the diagnosis of ocular infectious diseases. Semin Ophthalmol 34: 223-231, 2019.

10. Wang YE, Tepelus TC, Vickers LA, Baghdasaryan E, Gui W, Huang P, Irvine JA, Sadda S, Hsu HY and Lee OL: Role of in vivo confocal microscopy in the diagnosis of infectious keratitis. Int Ophthalmol 39: 2865-2874, 2019.

11. Lin A, Rhee MK, Akpek EK, Amescua G, Farid M, Garcia-Ferrer FJ, Varu DM, Musch DC, Dunn SP and Mah FS American Academy of Ophthalmology Preferred Practice Pattern Cornea and External Disease Panel. Bacterial keratitis preferred practice pattern ${ }^{\circledR}$. Ophthalmology 126: P1-P55, 2019.

12. Kaye SB, Rao PG, Smith G, Scott JA, Hoyles S, Morton CE, Willoughby C, Batterbury M and Harvey G: Simplifying collection of corneal specimens in cases of suspected bacterial keratitis. J Clin Microbiol 41: 3192-3197, 2003

13. Bourcier T, Thomas F, Borderie V, Chaumeil C and Laroche L: Bacterial keratitis: Predisposing factors, clinical and microbiological review of 300 cases. Br J Ophthalmol 87: 834-838, 2003.

14. Carnt N, Samarawickrama C, White A and Stapleton F: The diagnosis and management of contact lens-related microbial keratitis. Clin Exp Optom 100: 482-493, 2017.

15. Das S, Sharma S, Kar S, Sahu SK, Samal B and Mallick A: Is inclusion of Sabouraud dextrose agar essential for the laboratory diagnosis of fungal keratitis? Indian J Ophthalmol 58: 281-286, 2010

16. Robaei D, Chan UT, Khoo P, Cherepanoff S, Li YC, Hanrahan J and Watson S: Corneal biopsy for diagnosis of recalcitrant microbial keratitis. Graefes Arch Clin Exp Ophthalmol 256: 1527-1533, 2018

17. Benson WH and Lanier JD: Comparison of techniques for culturing corneal ulcers. Ophthalmology 99: 800-804, 1992.

18. Bharathi MJ, Ramakrishnan R, Meenakshi R, Mittal S, Shivakumar C and Srinivasan M: Microbiological diagnosis of infective keratitis: Comparative evaluation of direct microscopy and culture results. Br J Ophthalmol 90: 1271-1276, 2006.

19. Epley KD, Katz HR, Herling I and Lasky JB: Platinum spatula versus mini-tip culturette in culturing bacterial keratitis. Cornea 17: 74-78, 1998

20. Kaye S, Sueke H, Romano V, Chen JY, Carnt N, Tuft S and Neal T: Impression membrane for the diagnosis of microbial keratitis. Br J Ophthalmol 100: 607-610, 2016.

21. Sharma S, Kunimoto DY, Gopinathan U, Athmanathan S, Garg P and Rao GN: Evaluation of corneal scraping smear examination methods in the diagnosis of bacterial and fungal keratitis: A survey of eight years of laboratory experience. Cornea 21 643-647, 2002
22. Eleinen KG, Mohalhal AA, Elmekawy HE, Abdulbaki AM, Sherif AM, El-Sherif RH and Abdul Rahman EM: Polymerase chain reaction-guided diagnosis of infective keratitis-a hospital-based study. Curr Eye Res 37: 1005-1011, 2012.

23. Shimizu D, Miyazaki D, Ehara F, Shimizu Y, Uotani R, Inata K, Sasaki SI and Inoue Y: Effectiveness of 16S ribosomal DNA real-time PCR and sequencing for diagnosing bacterial keratitis. Graefes Arch Clin Exp Ophthalmol 258: 157-166, 2020.

24. Bhadange Y, Sharma S, Das S and Sahu SK: Role of liquid culture media in the laboratory diagnosis of microbial keratitis. Am J Ophthalmol 156: 745-751, 2013.

25. McLeod SD, Kumar A, Cevallos V, Srinivasan M and Whitcher JP: Reliability of transport medium in the laboratory evaluation of corneal ulcers. Am J Ophthalmol 140: 1027-1031, 2005.

26. Rodman RC, Spisak S, Sugar A, Meyer RF, Soong HK and Musch DC: The utility of culturing corneal ulcers in a tertiary referral center versus a general ophthalmology clinic. Ophthalmology 104: 1897-1901, 1997.

27. Alexandrakis G, Haimovici R, Miller D and Alfonso EC: Corneal biopsy in the management of progressive microbial keratitis. Am J Ophthalmol 129: 571-576, 2000.

28. Younger JR, Johnson RD, Holland GN, Page JP, Nepomuceno RL, Glasgow BJ, Aldave AJ, Yu F, Litak J and Mondino BJ; UCLA Cornea Service: Microbiologic and histopathologic assessment of corneal biopsies in the evaluation of microbial keratitis. Am J Ophthalmol 154: 512-519.e2, 2012.

29. Badiee P, Nejabat M, Alborzi A, Keshavarz F and Shakiba E: Comparative study of Gram stain, potassium hydroxide smear, culture and nested PCR in the diagnosis of fungal keratitis. Ophthalmic Res 44: 251-256, 2010.

30. Kim JH, Yum JH, Lee D and Oh SH: Novel technique of corneal biopsy by using a femtosecond laser in infectious ulcers. Cornea 27: 363-365, 2008.

31. Diamond J, Leeming J, Coombs G, Pearman J, Sharma A, Illingworth C, Crawford G and Easty D: Corneal biopsy with tissue micro-homogenisation for isolation of organisms in bacterial keratitis. Eye (Lond) 13: 545-549, 1999.

32. Brănişteanu DE, Pintilie A, Andreş LE, Dimitriu A, Oanţă A, Stoleriu G and Brănisteanu DC: Ethiopatogenic hypotheses in lichen planus. Rev Med Chir Soc Med Nat Iasi 120: 760-767, 2016.

33. Branisteanu DE, Pintilie A, Dimitriu A, Cerbu A, Ciobanu D, Oanta A and Tatu AL: Clinical, laboratory and therapeutic profile of lichen planus. Rev Med Chir Soc Med Nat Iasi 125: 25-32, 2017.

34. Brănişteanu DE, Ianoşi SL, Dimitriu A, Stoleriu G, Oanţă A and Brănisteanu DC: Drug-induced Rowell syndrome, a rare and difficult to manage disease: A case report. Exp Ther Med 15: 785-788, 2018.

35. Gupta N: DNA extraction and polymerase chain reaction. J Cytol 36: 116-117, 2019.

36. Zhao G, Zhai H, Yuan Q, Sun S, Liu T and Xie L: Rapid and sensitive diagnosis of fungal keratitis with direct PCR without template DNA extraction. Clin Microbiol Infect 20: O776-O782, 2014.

37. Simon C, Franke A and Martin A: The polymerase chain reaction: DNA extraction and amplification. In: Molecular Techniques in Taxonomy. Hewitt GM, Johnston AW and Young JP (eds). Vol 57. Springer, Berlin, pp329-355, 1991.

38. Embong Z, Wan Hitam WH, Yean CY, Rashid NH, Kamarudin B, Abidin SK, Osman S, Zainuddin ZF and Ravichandran M: Specific detection of fungal pathogens by $18 \mathrm{~S}$ rRNA gene PCR in microbial keratitis. BMC Ophthalmol 8: 7, 2008.

39. Tananuvat N, Salakthuantee K, Vanittanakom N, Pongpom M and Ausayakhun S: Prospective comparison between conventional microbial work-up vs PCR in the diagnosis of fungal keratitis. Eye (Lond) 26: 1337-1343, 2012

40. Itahashi M, Higaki S, Fukuda M and Shimomura Y: Detection and quantification of pathogenic bacteria and fungi using real-time polymerase chain reaction by cycling probe in patients with corneal ulcer. Arch Ophthalmol 128: 535-540, 2010.

41. Goldschmidt P, Degorge S, Che Sarria P, Benallaoua D, Semoun O, Borderie V, Laroche L and Chaumeil C: New strategy for rapid diagnosis and characterization of fungal infections: The example of corneal scrapings. PLoS One 7: e37660, 2012.

42. Karsenti N, Lau R, Purssell A, Chong-Kit A, Cunanan M, Gasgas J, Tian J, Wang A, Ralevski F and Boggild AK: Development and validation of a real-time PCR assay for the detection of clinical acanthamoebae. BMC Res Notes 10: 355, 2017. 
43. Li Z, Breitwieser FP, Lu J, Jun AS, Asnaghi L, Salzberg SL and Eberhart CG: Identifying corneal infections in formalin-fixed specimens using next generation sequencing. Invest Ophthalmol Vis Sci 59: 280-288, 2018.

44. Holmgaard DB, Barnadas C, Mirbarati SH, O'Brien Andersen L, Nielsen HV and Stensvold CR: Detection and identification of Acanthamoeba and other nonviral causes of infectious keratitis in corneal scrapings by real-time PCR and next-generation sequencing-based 16S-18S gene analysis. J Clin Microbiol 59: e02224-20, 2021.

45. Eguchi H, Hotta F, Kuwahara T, Imaohji H, Miyazaki C, Hirose M, Kusaka S, Fukuda M and Shimomura Y: Diagnostic approach to ocular infections using various techniques from conventional culture to next-generation sequencing analysis. Cornea 36 (Suppl 1): S46-S52, 2017.

46. Ren Z, Liu Q, Wang Y, Dong Y and Huang Y: Diagnostic information profiling and evaluation of causative fungi of fungal keratitis using high-throughput internal transcribed spacer sequencing. Sci Rep 10: 1640, 2020.

47. Vaddavalli PK, Garg P, Sharma S, Sangwan VS, Rao GN and Thomas R: Role of confocal microscopy in the diagnosis of fungal and Acanthamoeba keratitis. Ophthalmology 118: 29-35, 2011.
48. Erie JC, McLaren JW and Patel SV: Confocal microscopy in ophthalmology. Am J Ophthalmol 148: 639-646, 2009.

49. Goh JW, Harrison R, Hau S, Alexander CL, Tole DM and Avadhanam VS: Comparison of in vivo confocal microscopy, PCR and culture of corneal scrapes in the diagnosis of Acanthamoeba keratitis. Cornea 37: 480-485, 2018.

50. Kanavi MR, Javadi M, Yazdani S and Mirdehghanm S: Sensitivity and specificity of confocal scan in the diagnosis of infectious keratitis. Cornea 26: 782-786, 2007.

51. Su PY, Hu FR, Chen YM, Han JH and Chen WL: Dendritiform cells found in central cornea by in-vivo confocal microscopy in a patient with mixed bacterial keratitis. Ocul Immunol Inflamm 14: 241-244, 2006

52. Labbé A, Khammari C, Dupas B, Gabison E, Brasnu E, Labetoulle $\mathrm{M}$ and Baudouin $\mathrm{C}$ : Contribution of in vivo confocal microscopy to the diagnosis and management of infectious keratitis. Ocul Surf 7: 41-52, 2009. International (CC BY-NC-ND 4.0) License. 